\title{
Modelling the Additive Functional Equations through RSM Matrices
}

\author{
Pasupathi Narasimman ${ }^{1}$, K.Ravi ${ }^{2}$, Sandra Pinelas ${ }^{3}$ \\ ${ }^{1}$ Department of Mathematics, \\ Thiruvalluvar University College of Arts and Science, \\ Gazhalnayaganpatti, Tirupattur-635 901, TamilNadu, India. \\ drpnarasimman@gmail.com \\ 2 Department of Mathematics, Sacred Heart College, \\ Tirupattur - 635 601, TamilNadu, India. \\ shckravi@yahoo.co.in \\ ${ }^{3}$ Academia Militar, Departamento de Ciências Exactas e Naturais, \\ Av.Conde Castro Guimarães, 2720-113 Amadora, Portugal \\ sandra.pinelas@gmail.com
}

\section{ABSTRACT}

This paper suggests one possible method to model additive type of functional equations using eigenvalues and eigenvectors of matrices with suitable numerical examples. The authors have defined a new type of Row Sum Matrix(RSM) and have discussed its eigenvalues and eigenvectors in order to model functional equations. The famous additive Cauchy functional equation and logical functional equation have also been modelled using identity matrix and logical matrix in this study.

\section{Introduction}

The study of functional equations becomes essential as it provides a powerful approach to work with important concepts and relationships in analysis and algebra with regard to symmetry, linearity and equivalence. Though the systematic study of such equations is a relatively recent area of mathematical study, they have been considered earlier in various forms by mathematicians such as Euler in the $18^{\text {th }}$ century and Cauchy in the $19^{\text {th }}$ century.

A significant number of tools in today's Mathematics have been contributed by the theory of functional equations which is one of the growing branches of Mathematics. Many new applied problems and theories have motivated functional equations to develop new approaches and methods. D'Alembert, Euler, Gauss, Cauchy, Abel, Weierstrass, Darboux and Hilbert are among the great mathematicians who have been concerned with functional equations and methods of solving them.

Functional equations represent an alternative way of modelling problems in Physics. The interesting aspect in modelling physical problem through functional equation is that there is no necessity to assume the differentiability of the function $f$. Consequently, the functional equations lead often to other solutions than those given by partial differential equations, and these other solutions can be of interest to physicists. The most appealing characteristic of functional equation is its capacity to design mathematical models.

There have been so many researchers studying the solution and stability of different types of functional equations like additve, quadratic, cubic, quartic and mixed type of additive-quadratic, quadratic-cubic and so on. How ever, studies over the origin and formation of such functional equations are not convincing as they are only structure based trial and error methods. This fact has been influential for the authors to undergo a formal study on modelling functional equations.

The several functional equations and their stability problems have been extensively investigated by many mathematicians (see $[2,3,4,5,6,7,8,9,10,11,12,13,14,15,16,17,18,19,20,21,22,23]$ )

This paper is organised as follows: In section-2, authors discuss the preliminaries and definitions of functional equations. In section-3, authors introduce and discuss the new type of Row Sum Matrix (RSM) and propose a new method namely Pasupathi Narasimman method to model the additive functional equations through eigenvalues and eigenvectors of RSM. In section-4 and 5, authors model the most famous additive Cauchy functional equation and logical functional equation respectively using RSM. Finally, section-6 ends with conclusion.

\section{Preliminaries and Definition}

A Hungarian Mathematician J. Aczel [1], an excellent specialist in functional equations, defines the functional equation as follows:

\section{Definition 2.1 Functional Equation:}

Functional Equations are equations in which both sides are terms constructed from the finite number of unknown functions and a finite number of independent variables.

Example $2.2 f(2 x+y)+f(2 x-y)=8 f(x)+2 f(y)$. 


\section{Definition 2.3 Solutions of Functional Equation:}

A solution of a functional equation is a function which satisfies the equation.

Example 2.4 Cauchy Functional Equations

$$
\begin{aligned}
& f(x+y)=f(x)+f(y), \\
& f(x+y)=f(x) f(y), \\
& f(x y)=f(x)+f(y)
\end{aligned}
$$

have solutions $f(x)=k x, f(x)=e^{x}, f(x)=\ln x$, respectively.

\section{Additive Functional Equation corresponding to Row Sum Matrix \\ Definition 3.1 Row Sum Matrix(RSM)}

A square matrix is said to be row sum matrix, if it has each row sum values are equal. A RSM-2 matrix is the row sum matrix of order 2 .

Example 3.2 A general RSM-2 matrix is given by $\left(\begin{array}{ll}a_{11} & a_{12} \\ a_{21} & a_{22}\end{array}\right)$ where $a_{11}, a_{21} ; a_{12}, a_{22}$ are any integers with $a_{11}+a_{12}=a_{21}+a_{22}$.

Numerical Example $3.3\left(\begin{array}{cc}-2 & -4 \\ 1 & -7\end{array}\right)$

Note 3.4 The identity matrix and logical matrix of order 2 are the best example of RSM-2 matrix.

Lemma 3.5 If $A=\left(\begin{array}{ll}a_{11} & a_{12} \\ a_{21} & a_{22}\end{array}\right)$ be the RSM-2 matrix, then the eigenvalues of $A$ are

(i) $\lambda_{1}=a_{11}-a_{21}$,

(ii) $\lambda_{2}=a_{11}+a_{12}$.

Proof. If $A=\left(\begin{array}{ll}a_{11} & a_{12} \\ a_{21} & a_{22}\end{array}\right)$ be the RSM-2 matrix, then by the definition of RSM-2

$$
a_{11}+a_{12}=a_{21}+a_{22}
$$

The characteristic equation of matrix $A$ is $\left|\begin{array}{cc}a_{11}-\lambda & a_{12} \\ a_{21} & a_{22}-\lambda\end{array}\right|=0$, which gives

$$
\lambda^{2}-\left(a_{11}+a_{22}\right) \lambda+\left(a_{11} a_{22}-a_{21} a_{12}\right)=0 .
$$

Now, solving equation (2) by using (1), we get

$$
\left(\left(\lambda-\left(a_{11}-a_{21}\right)\right)\left(\lambda-\left(a_{11}+a_{12}\right)\right)\right)=0 .
$$

The equation (3) leads $\lambda_{1}=a_{11}-a_{21}$ and $\lambda_{2}=a_{11}+a_{12}$

Numerical Example 3.6 The RSM-2 matrix and its eigen values are

$$
\left(\begin{array}{cc}
-2 & 6 \\
1 & 3
\end{array}\right) ; \lambda_{1}=-3 \text { and } \lambda_{2}=4 \text {. }
$$


Remark 3.7 If $\lambda_{1}$ and $\lambda_{2}$ be the eigenvalues of RSM-2 matrix, then the difference between eigenvalues is equal to the sum of second diagonal elements in a matrix. That is $\lambda_{2}-\lambda_{1}=a_{12}+a_{21}$.

Numerical Example 3.8 For the RSM-2 matrix $\left(\begin{array}{ll}1 & 8 \\ 0 & 9\end{array}\right) ; \quad \lambda_{1}=1$ and $\lambda_{2}=9$, we note that $\lambda_{2}-\lambda_{1}=a_{12}+a_{21}=8$

The following remark gives the eigenvectors $X_{1}$ and $X_{2}$ corresponding to the eigenvalues $\lambda_{1}$ and $\lambda_{2}$ of the RSM-2 matrix.

Remark 3.9 For the RSM-2 matrix $A=\left(\begin{array}{ll}a_{11} & a_{12} \\ a_{21} & a_{22}\end{array}\right)$, its eigenvalues are $\lambda_{1}=a_{11}-a_{21}, \lambda_{2}=a_{11}+a_{12}$. Let $X_{1}$ and $X_{2}$ are eigenvectors of the RSM-2 matrix $A$ corresponding to the eigenvalues $\lambda_{1}$ and $\lambda_{2}$ respectively, then

$$
X_{1}=\left(\begin{array}{c}
a_{12} \\
-a_{21}
\end{array}\right), \quad X_{2}=\left(\begin{array}{c}
a_{11}+a_{12} \\
a_{11}+a_{12}
\end{array}\right) \text {. }
$$

Numerical Example 3.10 Consider the RSM-2 matrix $\left(\begin{array}{ll}1 & 5 \\ 4 & 2\end{array}\right)$. When $\lambda_{1}=-3$ the eigenvector is $X_{1}=\left(\begin{array}{c}5 \\ -4\end{array}\right)$. When $\lambda_{2}=6$ the eigenvector is $X_{2}=\left(\begin{array}{l}6 \\ 6\end{array}\right)$

Definition 3.11 Additive Matrix: A matrix is said to be additive matrix, if it gives the additive functional equation.

Definition 3.12 Quadratic Matrix: A matrix is said to be quadratic matrix, if it gives the quadratic functional equation.

Definition 3.13 Mixed Matrix: A matrix is said to be Mixed matrix, if it gives the Mixed type functional equation like additive-quadratic, quadratic-cubic, cubic-quartic, additive-quadratic-cubic, etc.

Remark 3.14 Possible Model Matrices for RSM-2: The possible model matrices for RSM-2 matrix $A=\left(\begin{array}{ll}a_{11} & a_{12} \\ a_{21} & a_{22}\end{array}\right)$ are $M_{1}=\left[\alpha X_{1}, \beta X_{2}\right]$ and $M_{2}=\left[\beta X_{2}, \alpha X_{1}\right]$, where $\alpha, \beta \in R$.

\section{Remark 3.15 Pasupathi Narasimman Method}

Let $X$ and $Y$ be the Vector spaces. Define a mapping $f: X \rightarrow Y$. Now, using $A$ and $M_{1}$ or $M_{2}$ we may model the following functional equation

$$
\begin{aligned}
& f\left(a_{11} x+m_{11} y\right)+f\left(a_{12} x+m_{12} y\right) \\
& =f\left(a_{21} x+m_{21} y\right)+f\left(a_{22} x+m_{22} y\right)+\alpha f\left(\left(\lambda_{2}-\lambda_{1}\right) y\right)
\end{aligned}
$$

for all $x, y \in X, \alpha \in R, \lambda_{1}$ and $\lambda_{2}$ are the eigenvalues of $A$.

It is easy to check that the functional equation (4) is additive for $\alpha \in R$. Hence, the RSM-2 matrices are additive matrices, since it gives additive functional equations.

Numerical Example 3.16 Let $B=\left(\begin{array}{ll}8 & 4 \\ 6 & 6\end{array}\right)$ be the RSM-2 matrix such that $\lambda_{1}=2$ and $\lambda_{2}=12$.

Here, authors select some of the following possible model matrices of $B$ using $M_{1}$ and $M_{2}$ to model the functional equations through (4). 


$$
\begin{aligned}
& N_{1}=\left(\begin{array}{cc}
4 & 12 \\
-6 & 12
\end{array}\right) \text { for } \alpha=1, \beta=1, \\
& N_{2}=\left(\begin{array}{cc}
2 & 12 \\
-3 & 12
\end{array}\right) \text { for } \alpha=\frac{1}{2}, \beta=1, \\
& N_{3}=\left(\begin{array}{cc}
-2 & 1 \\
3 & 1
\end{array}\right) \text { for } \alpha=-\frac{1}{2}, \beta=\frac{1}{12} \\
& N_{4}=\left(\begin{array}{cc}
1 & -2 \\
1 & 3
\end{array}\right) \text { for } \alpha=-\frac{1}{2}, \beta=\frac{1}{12}, \\
& N_{5}=\left(\begin{array}{cc}
1 & -1.333 \\
1 & 2
\end{array}\right) \text { for } \alpha=-\frac{1}{3}, \beta=\frac{1}{12} .
\end{aligned}
$$

Now, using $B$ and $N_{1}$ through (4), we may model the following functional equation

$$
\begin{aligned}
& f(8 x+4 y)+f(4 x+12 y) \\
& \quad=f(6 x-6 y)+f(6 x+12 y)+f(10 y)
\end{aligned}
$$

for all $x, y \in X$. Using $B$ and $N_{2}$ through (4), we may model the following functional equation

$$
\begin{aligned}
& f(8 x+2 y)+f(4 x+12 y) \\
& \quad=f(6 x-3 y)+f(6 x+12 y)+\frac{1}{2} f(10 y)
\end{aligned}
$$

for all $x, y \in X$. Using $B$ and $N_{3}$ through (4), we may model the following functional equation

$$
\begin{aligned}
& f(8 x-2 y)+f(4 x+y) \\
& \quad=f(6 x+3 y)+f(6 x+y)-\frac{1}{2} f(10 y)
\end{aligned}
$$

for all $x, y \in X$. Using $B$ and $N_{4}$ through (4), we may model the following functional equation

$$
\begin{aligned}
& f(8 x+y)+f(4 x-2 y) \\
& \quad=f(6 x+y)+f(6 x+3 y)-\frac{1}{2} f(10 y)
\end{aligned}
$$

for all $x, y \in X$. Using $B$ and $N_{5}$ through (4), we may model the following functional equation

$$
\begin{aligned}
& f(8 x+y)+f(4 x-1.333 y) \\
& \quad=f(6 x+y)+f(6 x+2 y)-\frac{1}{3} f(10 y)
\end{aligned}
$$

for all $x, y \in X$.

Clearlly the functional equations from (5) to (9) are additive, since $f(x)=x$ is the solution. Hence, the RSM-2 matrices are additive matrices.

\section{Modelling the Additive Cauchy Functional Equation}

In this section, authors model the additive Cauchy functional equation 


$$
f(x+y)=f(x)+f(y)
$$

which is most famous among the functional equations.

Consider RSM-2 matrix $I=\left(\begin{array}{ll}1 & 0 \\ 0 & 1\end{array}\right)$ a well known Identity matrix. Using $M_{1}$ given in Remark 3.14, we get the following model matrix for $I$.

$$
N_{9}=\left(\begin{array}{ll}
0 & 1 \\
0 & 1
\end{array}\right) \text { for } \alpha=1, \beta=1 .
$$

Using $M_{2}$ given in Remark 3.14, we get the following model matrix for $I$.

$$
N_{10}=\left(\begin{array}{ll}
1 & 0 \\
1 & 0
\end{array}\right) \text { for } \alpha=1, \beta=1 \text {. }
$$

Now, Using $I$ and $N_{9}$ or $N_{10}$ through (4) with assuming $f(0)=0$, one can model the famous Cauchy additive functional equation (10).

\section{Modelling the Additive Functional Equation corresponding to Logical Matrix \\ Definition 5.1 Logical matrix:}

A matrix whose entries are all either 0 or 1 .

In this section, authors model the additive functional equation corresponding to a matrix whose entries are all 1 which is of RSM-2 type. That is $L=\left(\begin{array}{ll}1 & 1 \\ 1 & 1\end{array}\right)$ a well known Logical matrix.

Using $M_{1}$ given in Remark 3.14, we get the following model matrix for $L$.

$$
N_{11}=\left(\begin{array}{cc}
1 & 2 \\
-1 & 2
\end{array}\right) \text { for } \alpha=1, \beta=1
$$

Using $M_{2}$ given in Remark 3.14, we get the following model matrix for $L$.

$$
N_{12}=\left(\begin{array}{cc}
2 & 1 \\
2 & -1
\end{array}\right) \text { for } \alpha=1, \beta=1
$$

Now, Using $L$ and $N_{11}$ or $N_{12}$ through (4) one can model the following additive functional equation

$$
f(x+2 y)+f(x+y)=f(x+2 y)+f(x-y)+f(2 y)
$$

for all $x, y \in X$. Which implies,

$$
f(x+y)-f(x-y)=f(2 y)
$$

for all $x, y \in X$. Here after, we may call the functional equation (12) is a Logical functional equation since it is derived from Logical matrix.

\section{Conclusion}

Thus, a new type of matrix RSM-2 is introduced and its eigenvalues and eigenvectors have been discussed and explained. We introduced a new and very first method called Pasupathi Narasimman method to model the additive type functional equations through eigenvalues and eigenvectors of matrices.

This is the first attempt to model additive functional equations using eigenvalues and eigenvectors of matrices.

A famous Cauchy functional equation (10) and Logical functional equation (12) have also been modelled using Identity matrix and Logical matrix of order 2 respectively with the proposed Pasupathi Narasimman method.

\section{Note 6.1 Open Problem}

The modelled additive functional equation (4) with RSM-2 matrix using the proposed Pasupathi Narasimman method in 
this study can be analyised in the future with Hyers-Ulam-Rassias stability and J.M.Rassias stability. This proposed open problem can lead to substantial contributions in the field of stability of functional equations and further developments in the field.

\section{References}

[1] J. Aczel and J. Dhombres, Functional Equation in Several Variables, Cambridge Univ. Press, 1989

[2] T. Aoki, On the stability of the linear transformation in Banach spaces, J. Math . Soc.Japan,2 (1950), 64-66.

[3] A. Bodaghi, I. A. Alias and M. H. Ghahramani, Approximately cubic functional equations and cubic multipliers, J. Inequal. Appl., Article No. 53, (2011).

[4] A. Bodaghi and I. A. Alias, Approximate ternary quadratic derivations on ternary Banach algebras and $C^{*}$-ternary rings, Adv. Differene Equa.,2012, Article No. 11, (2012).

[5] A. Bodaghi, I. A. Alias and M. H. Ghahramani, Ulam stability of a quartic functional equation, Abs. Appl. Anal. (2012), Art. ID 232630 (2012).

[6] I.S. Chang and H.M. Kim, On the Hyers-Ulam Stability of Quadratic Functional Equations, J. Inequal. Pure Appl. Math., 3 (2002), Art. 33.

[7] P.W.Cholewa, Remarks on the stability of functional equations, Aequationes Math. 27 (1984) 76-86.

[8] S. Czerwik, On the stability of the quadratic mapping in normed spaces, Abh. Math. Sem. Univ.Hamburg 62 (1992) 59 64.

[9] P.Gavruta, A generalization of the Hyers-Ulam-Rassias stability of approximately additive mappings, J. Math. Anal. Appl. 184 (1994) 431-436.

[10] D. H. Hyers, On the stability of the linear functional equation, Proc. Natl. Acad. Sci. 27 (1941) 222-224.

[11] K. W. Jun and H. M. Kim, The Generalized Hyers-Ulam-Rassias Stability of a Cubic Functional Equation, J. Math. Anal. Appl., 274 (2002), 867-878.

[12] PI. Kannappan, Quadratic functional equation and inner product spaces, Results Math. 27 (1995) 368-372.

[13] A.K.Mirmostafaee and M.S. Moslehian, Fuzzy almost quadratic functions, Results Math.doi:10.1007/s00025-0070278-9.

[14] Abbas Najati, The Generalized Hyers-Ulam-Rassias Stability of a Cubic Functional Equation, Turk J.Math, 31 (2007), pp. 395-408.

[15] K. H. Park and Y.S. Jung, Stability for a cubic functional equation, Bull. Korean Math. Soc., 41(2) (2004), 347-357.

[16] J.M. Rassias, On approximation of approximately linear mappings by linear mapping, J.Funct. Anal. 46 (1982),no. 1, 126-130.

[17] J.M. Rassias, On approximation of approximately linear mappings by linear mappings, Bull.Sci. Math. (2) 108 (1984), NO. 4, 445-446.

[18] J.M. Rassias, Hyers-Ulam stability of the quadratic functional equationin several variables, J. Ind. Math. Soc., 68 (2001), 65-73.

[19] J. M. Rassias, Solution of the Ulam stability problem for an Euler type quadratic functional equation, Southeast Asian Bull.Math., 26(2002), no. 1, 101-112.

[20] J.M. Rassias, On the General Quadratic Functional Equation, Bol.Soc. Mat. Mexicana, 11 (2005), 259 - 268.

[21] Matina J. Rassias, Generalized Hyers-Ulam Product-Sum Stability of a Cauchy Type Additive Functional Equation, European Journal of Pure and Applied Mathematics, Vol.4, No. 1, 2011, pp. 50-58.

[22] Th. M. Rassias, On the stability of the linear mapping in Banach spaces, Proc. Amer. Math. Soc. 72 (1978) $297-300$.

[23] K.Ravi J.M.Rassias, P.Narasimman, Stability of a cubic functional equation in fuzzy normed space, Journal of Applied Analysis and Computation, Volume 1, Number 3, August 2011, pp. 411-425. 